\title{
Danos qualitativos e quantitativos de Cinara atlantica (Wilson) (Hemiptera, Aphididae) em mudas de Pinus taeda Linnaeus (Pinaceae) ${ }^{1}$
}

\author{
Scheila R. M. Zaleski ${ }^{2}$, Sonia M. N. Lazzari $^{3} \&$ Susete do R. C. Penteado ${ }^{2,3}$ \\ ${ }^{1}$ Contribuição número 1517 do Departamento de Zoologia, Universidade Federal do Paraná. \\ ${ }^{2}$ Laboratório de Entomologia, Embrapa Florestas. Estrada da Ribeira, Km 111, 83411-000 Colombo, Paraná, Brasil. \\ E-mail:scheila_zaleski@hotmail.com; susete@cnpf.embrapa.br \\ ${ }^{3}$ Departamento de Zoologia, Universidade Federal do Paraná. Caixa Postal 19020, 81531-980 Curitiba, Paraná, Brasil. \\ E-mail: lazzari@ufpr.br
}

\begin{abstract}
Qualitative and quantitative damage by Cinara atlantica (Wilson) (Hemiptera, Aphididae) on Pinus taeda Linnaeus seedlings (Pinaceae). The giant conifer aphid, Cinara atlantica (Wilson, 1919) (Hemiptera, Aphididae), is one of the main insect pests on pine reforestation areas in Southern Brazil, nowadays. Since there is very few information about its damage, the objective of this study was to characterize and quantify the damage of $C$. atlantica on Pinus taeda. The experiment was carried out in a greenhouse, using a random design, with three infestation levels: $O$ (control), $10 \pm 2,20 \pm 2$ and $50 \pm 2$ aphids on each P. taeda seedling of 90 and 150 days old, with 20 replicates/treatment. The main damage observed were stunting, twisting, oversprouting and large amount of sooty mold on honeydew. The basal diameter was significantly reduced by $16.1 \%$ under $50 \pm 2$ aphids/plant of 90 days compared to the control. For the 150 days old seedlings, there was no statistical difference between the infestation levels. The plant height reduction was of $24.4 \%$ for the 90 days old plants with $50 \pm 2$ aphids and $32.6 \%$ for the 150 days old with $20 \pm 2$ aphids.
\end{abstract}

KEY WORDS. Aphid damages, giant conifer aphid, infestation level, loblolly pine.

RESUMO. O pulgão-gigante-do-pinus, Cinara atlantica (Wilson, 1919) (Hemiptera, Aphididae), é considerado atualmente uma das principais pragas dos reflorestamentos de pinus no Brasil. Como a informação sobre os danos causados por esta praga é restrita, esta pesquisa teve como objetivo caracterizar e quantificar os danos de $C$. atlantica em Pinus taeda. O experimento foi desenvolvido em casa de vegetação, seguindo um delineamento inteiramente casualizado. Foram testados três níveis de infestação com densidades populacionais de zero (testemunha protegida com inseticida) $10 \pm 2,20 \pm 2$ e $50 \pm 2$ afídeos por muda de $P$. taeda com idades de 90 e 150 dias. As mudas atacadas apresentaram redução no desenvolvimento, entortamento do fuste, superbrotação e presença abundante de fumagina devido ao desenvolvimento de fungo sobre o honeydew eliminado pelos afídeos. O diâmetro do colo diminuiu significativamente em $16,1 \%$ na densidade de $50 \pm 2$ afídeos/planta, nas mudas com 90 dias, quando comparado à testemunha. Nas mudas com 150 dias não houve diferença significativa no diâmetro do colo entre as parcelas infestadas e a testemunha. Houve uma redução de $24,4 \%$ na altura das mudas de 90 dias com a densidade de $50 \pm 2$ afídeos e $32,6 \%$ nas mudas de 150 dias, com intensidade de $20 \pm$ 2 afídeos.

PALAVRAS CHAVE. Danos de afídeos, pulgão-gigante-do-pinus, nível de infestação, mudas de pinus.

Pinus Linnaeus, 1754 (Pinaceae) é um gênero exótico de coníferas no Brasil, com cerca de dois milhões de hectares plantados, dos quais, 1,2 milhões estão localizados na região Sul e estado de São Paulo, compostos principalmente por Pinus elliottii Engelm e Pinus taeda Linnaeus (SHImizu \& Higa 1981). A intensificação da monocultura e a utilização de práticas silviculturais inadequadas têm tornado os plantios de Pinus suscetíveis ao estabelecimento e dispersão de pragas introduzidas.
O pulgão-gigante-do-pinus, Cinara atlantica (Wilson, 1919) (Hemiptera, Aphididae, Lachninae, Cinarini), originário da América do Norte (EASTOP 1972, JOHNSON \& LYON 1976), foi registrado no Brasil em 1998, observado inicialmente em plantios de $P$. taeda e $P$. elliottii, no município de Lages, estado de Santa Catarina, juntamente com colônias de Cinara pinivora (Wilson, 1919). Atualmente, C. atlantica está presente nos estados do Rio Grande do Sul, Santa Catarina e Paraná, em P. taeda, P. elliottii e 
Pinus patula Schiede \& Deppe, em Minas Gerais e São Paulo ocorre também em espécies de Pinus tropicais (Penteado et al. 2000).

Essas espécies de Cinara Curtis, 1835, descritas por WILSON (1919), formam colônias extensas nas plantas de pinus, causando danos diversos, incluindo o desenvolvimento da fumagina sobre o honeydew eliminado pelos pulgões, reduzindo a área fotossintética e dificultando os processos de respiração e transpiração da planta (PenteAdo et al. 2000).

Pepper \& Tissot (1973) observaram densas colônias de $C$. pinivora praticamente sobre toda a planta. Na Flórida e Carolina do Sul, foi observada a presença das colônias na ponta dos ramos; algumas vezes, foram encontrados afídeos dispersos ao longo de ramos mortos e sob acúmulo de acículas mortas presas nos ramos das árvores.

Segundo KIDD (1988), os danos podem ser causados pela extração dos nutrientes, da injeção de toxinas contidas nas secreções salivares, ou pela fumagina que se desenvolve sobre o honeydew eliminado pelos pulgões. Pode-se observar também, algumas modificações morfológicas próximas ao local de alimentação, como: afilamento irregular do tronco, dilatação nodal e rompimento da casca, todos eles contribuindo para a redução do valor econômico da madeira.

De acordo com Kfir et al. (1985) e Mills (1990), os adultos e as ninfas de Cinara sugam a seiva do floema da planta hospedeira, causando dessecação dos tecidos e, em altas densidades populacionais, provocam queda das acículas e até a morte da planta.

Fox \& GRIFFTH (1977) observaram uma redução significativa no crescimento em diâmetro e altura de $P$. taeda de um a dois anos de idade, infestada por C. atlantica, na Carolina do Sul. Os ataques mais intensos e com danos mais significativos são registrados, usualmente, em árvores jovens ou em viveiros (РАтTI \& Fox 1981). Kidd \& Tozer (1984) reforçam que esta espécie apresenta crescimento, sobrevivência e fecundidade ótimos quando ataca plantas jovens, em pleno crescimento, ou partes jovens de plantas adultas.

Em áreas onde se alternam períodos úmidos e secos, as colônias de Cinara sp. tendem a ser mais abundantes e causar maiores danos durante a estação seca (Ciesla 1991). Pepper \& TissoT (1973) verificaram que o pico populacional de C. pinivora ocorre entre o final de outono e durante o inverno, e praticamente desaparecem nos meses mais quentes, no leste dos Estados Unidos. No Brasil, Penteado et al. (2000) observaram que sua ocorrência também é maior nos meses mais frios, mas que, em dias de temperaturas mais elevadas, os afídeos desaparecem da parte aérea e abrigam-se nas raízes, em ninhos de formigas associadas. Essas formigas utilizam-se do honeydew e, ao mesmo tempo, realizam uma limpeza do local facilitando o desenvolvimento e locomoção dos pulgões.

Devido às notáveis infestações e danos causados por Cinara spp. em reflorestamentos de Pinus e à carência de estudos sobre estes afídeos no Brasil, foi realizada esta pesquisa, visando quantificar e caracterizar os danos de C. atlantica.

\section{MATERIAL E MÉTODOS}

Esta pesquisa foi desenvolvida em casa de vegetação na Embrapa Florestas, em Colombo, Paraná, no período de maio a novembro de 2002.

Foram selecionadas 160 mudas de tubete com aproximadamente o mesmo diâmetro de colo e altura, medidos, respectivamente com paquímetro e régua. As mudas foram inspecionadas sob microscópio estereoscópico, com aumento de 40 vezes, para certificar-se da ausência de pulgões, em seguida foram lavadas com hipoclorito a $1 \%$ e transplantadas para sacos plásticos médios (30 cm de altura x $10 \mathrm{~cm}$ diâmetro), contendo como substrato terra adubada (4-30-13). As mudas foram colocadas em casa de vegetação, distribuídas conforme o delineamento inteiramente casualizado, com oito tratamentos e 20 repetições, representadas por uma planta.

Os tratamentos foram os seguintes: 1) testemunha, plantas com 90 dias de idade e densidade populacional de 0 (zero) afídeos; 2) plantas com 90 dias de idade e $10 \pm 2$ afídeos; 3) plantas com 90 dias de idade e $20 \pm 2$ afídeos; 4) plantas com 90 dias de idade e $50 \pm 2$ afídeos; 5) testemunha, plantas com 150 dias de idade e 0 (zero) afídeos; 6) plantas com 150 dias de idade e $10 \pm 2$ afídeos; 7) plantas com 150 dias de idade e $20 \pm$ 2 afídeos; 8) plantas com 150 dias de idade e $50 \pm 2$ afídeos.

A temperatura média na casa de vegetação era $23^{\circ} \mathrm{C}$ e a umidade relativa era $69 \%$, no período. Os pulgões utilizados no experimento foram obtidos de ramos de Pinus coletados no campo e examinados no laboratório para a retirada dos insetos com um pincel fino. As mudas eram infestadas, preferencialmente, com ninfas a partir do terceiro instar e fêmeas ápteras. As mudas eram examinadas semanalmente para manter a densidade populacional de acordo com os tratamentos. As avaliações foram realizadas mensalmente durante um período de seis meses, medindo-se o diâmetro e a altura de todas as plantas com o auxílio de um paquímetro digital e uma trena. Os dados foram correlacionados com a densidade populacional e a idade das mudas.

Para a caracterização dos danos foram realizadas observações mensais de alterações morfológicas das plantas como perda da dominância apical, superbrotamento, amarelecimento das acículas e entortamento do fuste.

As plantas testemunhas foram pulverizadas com Acefate (Orthene) a cada 30 dias, na dosagem recomendada pelo fabricante (1g/L de água), para mantê-las completamente livres de infestação.

Os dados foram tabulados e os resultados submetidos às análises de regressão, de variância (ANOVA) e à comparação de médias pelo teste de Tukey a 5\% de probabilidade.

\section{RESULTADOS E DISCUSSÃO}

\section{Caracterização dos danos}

As mudas infestadas com C. atlantica, independente do nível de infestação, apresentaram alterações visuais na sua morfologia, como redução no desenvolvimento, entortamento do 

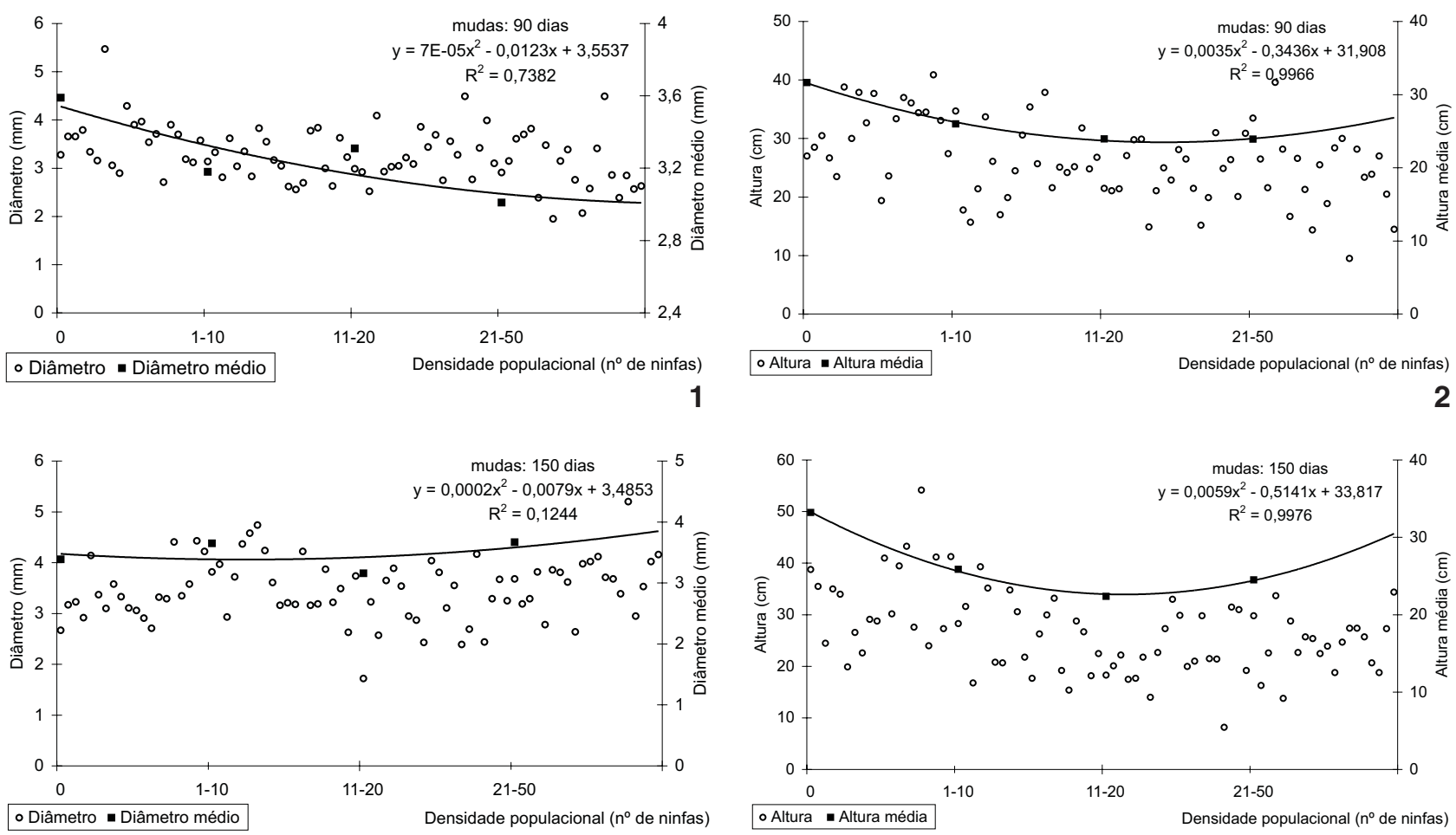

3

Figuras 1-4. Crescimento no diâmetro de colo e altura das mudas de Pinus taeda com: (1-2) 90 e (3-4)150 dias de idade e após seis meses de exposição a diferentes densidades populacionais de Cinara atlantica em casa de vegetação, temperatura média de $23^{\circ} \mathrm{C}$ e umidade média do ar de 69\%. Colombo, Paraná, 2002.

fuste e superbrotação, quando comparadas com as plantas testemunhas. Também, observou-se a presença de fumagina nas partes da planta onde havia infestação, corroborando com as observações de Penteado et al. (2000) referentes ao ataque de $C$. pinivora sobre espécies de Pinus no Sul do Brasil. KIDD (1988) afirmou que os distúrbios de crescimento da planta resultam da extração da seiva do floema no processo de alimentação desses insetos.

\section{Quantificação dos danos}

Após um período de seis meses de observações, verificouse que nas mudas de 90 dias de idade, o diâmetro médio de colo dos tratamentos com zero, $10 \pm 2$ e $20 \pm 2$ afídeos, não diferiram estatisticamente entre si. Porém, estes diferiram estatisticamente do tratamento com $50 \pm 2$ afídeos, com redução de $16,2 \%$ no diâmetro de colo (Fig. 1 e Tab. I). Nas mudas de 150 dias de idade apenas o tratamento com $20 \pm 2$ afídeos diferiu da testemunha e dos demais, sofrendo uma redução de apenas $6,8 \%$ (Fig. 2 e Tab. I).

Com relação à altura das mudas de $P$. taeda, verificou-se que nas mudas de 90 dias sem infestação, o crescimento foi estatisticamente maior $(31,6 \mathrm{~cm})$ do que nos demais tratamentos. Nas plantas infestadas ocorreu uma redução da altura pro- porcional à intensidade dos três níveis de infestação: 25,9 cm; $24,0 \mathrm{~cm}$ e $23,9 \mathrm{~cm}$, representando, respectivamente uma redução de $17,9 \%$, 24,3\% e 24,4\% (Fig. 3 e Tab. II). Apesar de ter ocorrido uma redução aparente em termos absolutos, a análise estatística mostrou que não houve diferença significativa entre os tratamentos. Contudo, se a redução deste parâmetro se mantiver até a idade de corte da planta, o dano será refletido no rendimento, com uma redução econômica considerável, independente do resultado do teste estatístico.

Da mesma forma, a altura das mudas de 150 dias sem infestação foi estatisticamente maior $(33,2 \mathrm{~cm})$ do que nas parcelas infestadas, e este parâmetro sofreu redução à medida que o nível de infestação aumentou. Nos tratamentos com $10 \pm 2$ afídeos a redução foi de $22,1 \%$; com $20 \pm 2$ afídeos foi de $32,6 \%$ e $50 \pm 2$ afídeos, de $26,2 \%$, porém, estatisticamente não apresentou diferenças significativas entre os tratamentos (Fig. 4 e Tab. II).

Esses resultados concordam com Fox \& Griffith (1977) que avaliaram os danos causados por C. atlantica em árvores de $P$. taeda de um a três anos, em casa de vegetação, através da medição de diâmetro e altura das plantas, e também concluíram que estes afídeos causam uma redução significativa no diâmetro de colo e na altura das plantas. Já para KATERERE (1982), as densidades altas e baixas do afídeo Eulachnus ryleyi (Williams, 
Tabela I. Diâmetro de colo e altura (média ( $s$ ) das mudas de Pinus taeda com 90 dias de idade e após seis meses de exposição a diferentes densidades populacionais de Cinara atlantica em casa de vegetação $\left(\mathrm{n}=20\right.$ plantas), com temperatura média de $23^{\circ} \mathrm{C}$ e umidade média do ar de 69\%. Colombo, Paraná, 2002.

\begin{tabular}{|c|c|c|c|c|c|c|c|c|}
\hline \multirow{3}{*}{ Tratamento } & \multicolumn{4}{|c|}{ Diâmetro de colo $(\mathrm{mm})$} & \multicolumn{4}{|c|}{ Altura $(\mathrm{cm})$} \\
\hline & \multicolumn{2}{|c|}{ Idade } & \multirow{2}{*}{$\begin{array}{c}\text { Crescimento } \\
\mathrm{x} \pm \mathrm{s}\end{array}$} & \multirow{2}{*}{ Proporção de crescimento } & \multicolumn{2}{|c|}{ Idade } & \multirow{2}{*}{$\frac{\text { Crescimento }}{x \pm s}$} & \multirow{2}{*}{ Proporção de crescimento } \\
\hline & 3 & 9 & & & 3 & 9 & & \\
\hline Testemunha & 2,50 & 6,09 & $3,59 \pm 0,6 a$ & 2,44 & 15,17 & 46,83 & $31,65 \pm 5,8 a$ & 3,09 \\
\hline $10 \pm 2$ & 2,59 & 5,77 & $3,18 \pm 0,4 a b$ & 2,23 & 13,59 & 39,58 & $25,99 \pm 6,3 b$ & 2,91 \\
\hline $20 \pm 2$ & 2,79 & 6,10 & $3,31 \pm 0,5 a b$ & 3,31 & 14,31 & 38,27 & $23,96 \pm 4,8 b$ & 2,67 \\
\hline $50 \pm 2$ & 2,81 & 6,48 & $3,01 \pm 0,6 b$ & 3,67 & 15,18 & 39,70 & $23,91 \pm 7,0 \mathrm{~b}$ & 2,61 \\
\hline
\end{tabular}

Médias seguidas pela mesma letra nas colunas não diferem significativamente entre si pelo teste de Tukey a 5\% de probabilidade.

Tabela II. Diâmetro de colo e altura (média ( s) das mudas de Pinus taeda com 150 dias de idade e após seis meses de exposição a diferentes densidades populacionais de Cinara atlantica em casa de vegetação $\left(n=20\right.$ plantas), com temperatura média de $23^{\circ} \mathrm{C}$ e umidade média do ar de 69\%. Colombo, Paraná, 2002.

\begin{tabular}{|c|c|c|c|c|c|c|c|c|}
\hline \multirow{3}{*}{ Tratamento } & \multicolumn{4}{|c|}{ Diâmetro de colo $(\mathrm{mm})$} & \multicolumn{4}{|c|}{ Altura $(\mathrm{cm})$} \\
\hline & \multicolumn{2}{|c|}{ Idade } & \multirow{2}{*}{$\begin{array}{c}\text { Crescimento } \\
x \pm s\end{array}$} & \multirow{2}{*}{ Proporção de crescimento } & \multicolumn{2}{|c|}{ Idade } & \multirow{2}{*}{$\frac{\text { Crescimento }}{x \pm s}$} & \multirow{2}{*}{ Proporção de crescimento } \\
\hline & 5 & 11 & & & 5 & 11 & & \\
\hline Testemunha & 3,32 & 6,71 & $3,39 \pm 0,5 a b$ & 2,02 & 18,90 & 52,12 & $33,22 \pm 8,6 a$ & 2,76 \\
\hline $10 \pm 2$ & 3,69 & 7,34 & $3,65 \pm 0,6 a$ & 1,99 & 19,58 & 45,47 & $25,89 \pm 7,0 \mathrm{~b}$ & 2,32 \\
\hline $20 \pm 2$ & 3,64 & 6,81 & $3,16 \pm 0,6 b$ & 1,87 & 18,19 & 40,60 & $22,40 \pm 6,3 b$ & 2,23 \\
\hline $50 \pm 2$ & 3,57 & 6,57 & $3,67 \pm 0,5 a$ & 1,84 & 18,54 & 42,45 & $24,52 \pm 5,3 b$ & 2,29 \\
\hline
\end{tabular}

Médias seguidas pela mesma letra nas colunas não diferem significativamente entre si pelo teste de Tukey a $5 \%$ de probabilidade.

1843) sobre $P$. patula não apresentaram diferenças significativas nos danos causados.

Com os valores de altura e diâmetro do colo obtidos, calculou-se a proporção de crescimento para os tratamentos. Para as plantas com 90 dias obteve-se, seis meses após, para a altura, valores de 3,1 (tratamento com $50 \pm 2$ afídeos) e 2,6 (testemunha) e para o diâmetro, 2,4 (tratamento com $50 \pm 2$ afídeos) e 3,7 (testemunha). Para as plantas de 150 dias, após seis meses, foram registrados valores para a altura de 2,8 (50 \pm 2 afídeos) e 2,3 e para o diâmetro, valores de 2,0 (50 \pm 2 afídeos) e 1,8 (testemunha). Estes resultados foram semelhantes aos obtidos por E.T. IEDE (com. pess.), o qual avaliou os danos provocados por C. atlantica na altura e diâmetro de plantas, em plantios de pinus. No primeiro deles, em uma área de $P$. taeda com 13 meses de idade, não houve diferença significativa na altura e diâmetro entre a testemunha (área protegida com inseticida) e a área desprotegida. A mesma tendência foi observada em outra área, de Pinus oocarpa Schiede \& Schelectendahl, com 15 meses de idade. Já numa terceira área, de $P$. taeda, avaliada com 16 meses, foi constatada diferença significativa na altura entre $o$ bloco tratamento $(3,8)$ e o bloco protegido com inseticida $(5,3)$, sendo que o diâmetro não apresentou diferença significativa entre as áreas.

Com base nesses resultados, pode-se concluir que, quando a densidade populacional de $C$. atlantica aumenta, há uma redução no crescimento em altura e diâmetro das mudas. Tam- bém os danos morfológicos devem ser considerados, pois, dependendo da intensidade do ataque, podem ser irrecuperáveis. Desta forma, devem ser definidas medidas de controle para evitar prejuízos econômicos e sociais no setor madeireiro.

\section{AGRADECIMENTOS}

À Coordenação de Aperfeiçoamento de Pessoal de Nível Superior (Capes), pela bolsa de estudo de mestrado concedida ao primeiro autor; e ao pesquisador Dr. Edilson Oliveira da Embrapa florestas pelo apoio na análise dos dados.

\section{REFERÊNCIAS BIBLIOGRÁFICAS}

Ciesla, W.M. 1991. The cypress aphid, Cinara cupressi (Buckton) in Africa, p. 33-47. In: W.M. CIESLA (Ed.). Exotic aphids pests of conifers - a crisis in African forestry. Muguga, Proceedings Kenya Forestry research Institute/FAO, VII+160p.

EAstop, V.F. 1972. A taxonomic review of the species of Cinara Curtis occurring in Britain (Hemiptera: Aphididae). Bulletin of the British Museum (Natural History) Entomology, London, 27 (2): 101-186.

Fox, R.C. \& K.H. GRIFFITH. 1977. Pine seedling growth loss caused by cinaran aphids in South Carolina. Journal of the Georgia Entomological Society, Tifton, 12 (1): 29-34.

Johnson, W.T. \& H.H. Lyon. 1976. Aphids on conifers, p. 68-69. In: W.T. JOHNSON \& H.H. LYON (Eds). Insects that feed on

Revista Brasileira de Zoologia 22 (3): 591-595, setembro 2005 
trees and shrubs - An Illustrated Practical guide. New York, Cornell University Press, 464p.

Katerere, Y. 1982. Aphid density and animal damage to Pinus patula Schiede and Deppe in a clone bank at Melsetter forest research station. South African Forestry Journal, Zimbabwe, 122 (3): 63-65.

KIDD, N.A.C. 1988. The large pine aphid on Scots pine in Britain, p. 111-128. In: A.A. BERRYMAN (Ed). Dynamics of forest insect populations. New York, Plenum Press, XXVII+603p.

KIDD, N.A.C. \& D.J. TozER. 1984. Host plant and crowding effects in the induction of alatae in the large pine aphid, Cinara pinea. Entomologia Experimentalis et Applicata, Dordrecht, 35 (2): 37-42.

Kfir, R.; F. Kirsten \& N.J. van Rensburg. 1985. Pauesia sp. (Hymenoptera: Aphididae), a parasite introduced into South Africa for biological control of the black pine aphid Cinara cronartii (Homoptera: Aphididae). Environmental Entomology, Colege park, 14 (5): 597-601.

Mills, N.J. 1990. Biological control of forest aphid pests in Afri- ca. Bulletin of Entomological Research, London, 80: 31-36. Patti, J.H. \& F.C. Fox. 1981. Seasonal occurrence of Cinara spp. and Essigella pini Wilson on loblolly pine, Pinus taeda L. Journal of the Georgia Entomological Society, Tifton, 16 (1): 96-105, 214-218.

Penteado, S.R.C.; R.F.Trentini; E.T. Iede; W. Reis Filho. 2000. Ocorrência, distribuição, danos e controle de pulgões do gênero Cinara em Pinus spp. no Brasil. Revista Floresta, Curitiba, 30 (1): 55-64.

Peper, J.O.; A.N. Tissot. 1973. Pine feeding species of Cinara in the Eastern U.S. (Homoptera: Aphididae). Florida Agricultural Experiment Station Monograph Series, Gainesville, 3: 1-160.

Shimizu, J.Y. \& A.R. Higa. 1981. Variação racial do P. taeda L. no Sul do Brasil até o sexto ano de idade. Boletim de Pesquisa Florestal, Curitiba, 2: 1-25.

WiLSON, H.F. 1919. Some new Lachnids of the genus Lachniella (Homoptera: Hemiptera). The Canadian Entomologist, Ottawa, 51 (1-2): 18-22, 41-47.

Recebido em 21.IX.2004; aceito em 26.VII.2005. 